Environmental Protection Department Operations and Regulatory Affairs Division

\title{
ChemTrack System Architecture
}

June 1997

Lawrence Livermore National Laboratory University of California, Livermore, California 94551 
This report has been reproduced directly from the best available copy.

Available to DOE and DOE contractors from the Office of Scientific and Technical Information P.O. Box 62, Oak Ridge, TN 37831

Prices available from (615) 576-8401, FTS 626-8401

Available to the public from the National Technical Information Service

U.S. Department of Commerce 5285 Port Royal Rd., Springfield, VA 22161 


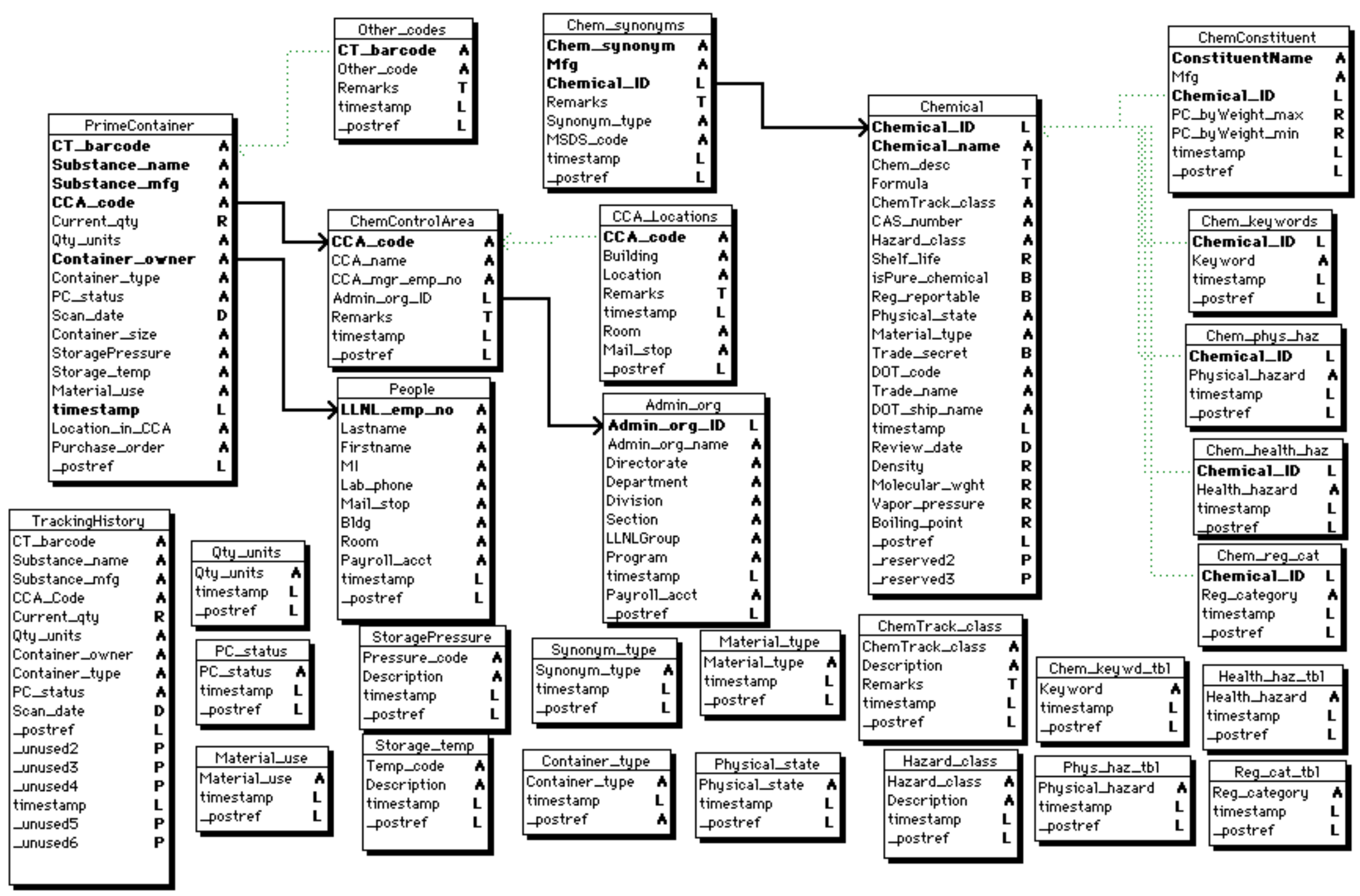




\begin{tabular}{|ll|}
\hline \multicolumn{2}{|c|}{-transaction } \\
\hline transaction_no & $\mathbf{L}$ \\
post_state & $\mathbf{I}$ \\
post_timestamp & $\mathbf{L}$ \\
retoode & $\mathbf{I}$ \\
retmsg & $\mathbf{T}$ \\
fail_stmint_no & $\mathbf{L}$ \\
\end{tabular}

\begin{tabular}{|ll|}
\hline \multicolumn{2}{|c|}{ statement } \\
\hline statement_no & L \\
transaction_no & L \\
file_no & I \\
postref_field & I \\
where_olause & T \\
reo_timestamp & L \\
local_key_val & L \\
local_key_fld & I \\
\hline
\end{tabular}

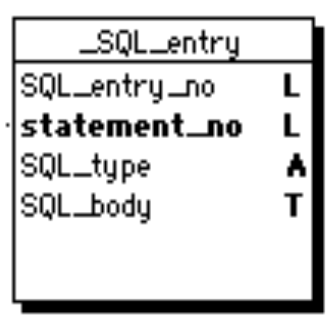

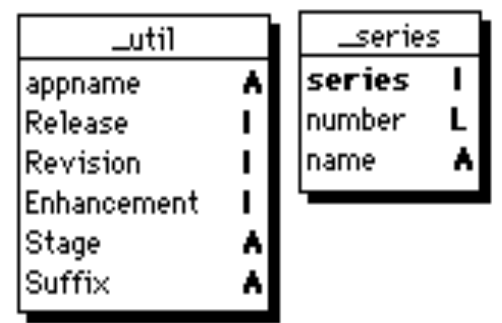

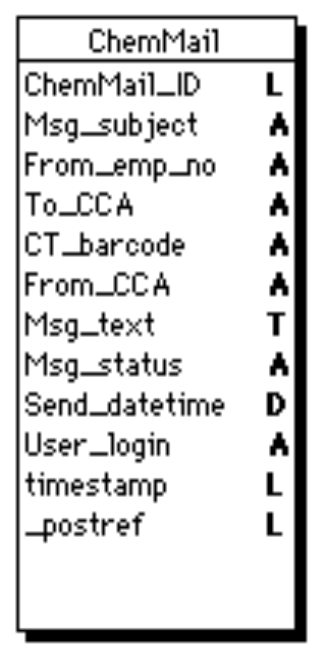

\begin{tabular}{|ll|}
\hline \multicolumn{1}{|c|}{ Inventory } \\
\hline CCA_code & A \\
CT_baroode & A \\
Inventory_date & D \\
Uplojd_date & D \\
ErrCode & L \\
Error_msg & T \\
\hline
\end{tabular}




\begin{tabular}{|l|l|l|}
\hline \multicolumn{2}{|l|}{ Structure:_util } \\
\hline appname & Alpha 20 & Enterable; Modifiable \\
Release & Integer & Enterable; Modifiable \\
Revision & Integer & Enterable; Modifiable \\
Enhancement & Enterable; Modifiable \\
Stage & Integer & Enterable; Modifiable \\
Suffix & Alpha 12 & Enterable; Modifiable \\
ServerPath & Alpha 20 & Enterable; Modifiable \\
\hline
\end{tabular}

\begin{tabular}{|l|l|l|}
\hline \multicolumn{2}{|c|}{ Structure:_series } \\
\hline $\begin{array}{l}\text { series } \\
\text { number } \\
\text { name }\end{array}$ & $\begin{array}{l}\text { Integer } \\
\text { Long Integer } \\
\text { Alpha 32 }\end{array}$ & $\begin{array}{l}\text { Indexed; Enterable; Modifiable } \\
\text { Enterable; Modifiable } \\
\text { Enterable; Modifiable }\end{array}$ \\
\hline
\end{tabular}




\section{Structure: PrimeContainer}

\begin{tabular}{l|l|l}
\hline CT_barcode & Alpha 10 & Indexed; Unique; Mandatory; Enterable \\
Substance_name & Alpha 80 & Indexed; Enterable; Modifiable \\
Substance_mfg & Alpha 40 & Indexed; Enterable; Modifiable \\
CCA_code & Alpha 15 & Indexed; Enterable; Modifiable \\
Current_qty & Real & Enterable; Modifiable \\
Qty_units & Alpha 10 & Enterable; Modifiable \\
Container_owner & Alpha 10 & Indexed; Enterable; Modifiable \\
Container_type & Alpha 10 & Enterable; Modifiable \\
PC_status & Alpha 12 & Enterable; Modifiable \\
Scan_date & Date & Enterable; Modifiable \\
Container_size & Alpha 10 & Enterable; Modifiable \\
StoragePressure & Alpha 2 & Enterable; Modifiable \\
Storage temp & Alpha 2 & Enterable; Modifiable \\
Material_use & Alpha 30 & Enterable; Modifiable \\
timestamp & Long Integer & Indexed; Enterable; Modifiable \\
Location_in_CCA & Alpha 40 & Enterable; Modifiable \\
Purchase_order & Alpha 20 & Enterable; Modifiable \\
postref & Long Integer & Enterable; Modifiable \\
\hline
\end{tabular}

\begin{tabular}{|l|l|l}
\hline \multicolumn{2}{|c}{ Structure: Chem_synonyms } \\
\hline Chem_synonym & Alpha 80 & Indexed; Mandatory; Enterable \\
Mfg & Alpha 40 & Indexed; Mandatory; Enterable \\
Chemical_ID & Long Integer & Indexed; Enterable; Modifiable \\
Remarks & Text & Enterable; Modifiable \\
Synonym_type & Alpha 20 & Enterable; Modifiable \\
MSDS code & Alpha 20 & Enterable; Modifiable \\
timestamp & Long Integer & Enterable; Modifiable \\
postref & Long Integer & Enterable; Modifiable \\
\hline
\end{tabular}




\begin{tabular}{|l|l|l|}
\hline \hline \multicolumn{2}{|c|}{ Structure: Other_codes } \\
\hline CT_barcode & Alpha 20 & Indexed; Enterable; Modifiable \\
Other code & Alpha 80 & Enterable; Modifiable \\
Remarks & Text & Enterable; Modifiable \\
timestamp & Long Integer & Enterable; Modifiable \\
_postref & Long Integer & Enterable; Modifiable \\
\hline
\end{tabular}

\begin{tabular}{|l|l|l|}
\hline \multicolumn{2}{|c|}{ Structure: Chemical } \\
\hline Chemical_I D & Long Integer & Indexed; Unique; Mandatory; Enterable \\
Chemical_name & Alpha 80 & Indexed; Enterable; Modifiable \\
Chem_desc & Text & Enterable; Modifiable \\
Formula & Text & Enterable; Modifiable \\
ChemTrack_class & Alpha 2 & Enterable; Modifiable \\
CAS_number & Alpha 11 & Enterable; Modifiable \\
Hazard_class & Alpha 2 & Enterable; Modifiable \\
Shelf_life & Real & Enterable; Modifiable \\
isPure_chemical & Eoolean & Enterable; Modifiable \\
Reg_reportable & Boolean & Enterable; Modifiable \\
Physical_state & Alpha 7 & Enterable; Modifiable \\
Material type & Alpha 20 & Enterable; Modifiable \\
Trade_secret & Boolean & Enterable; Modifiable \\
DOT_code & Enterable; Modifiable \\
Trade_name & Alpha 80 & Enterable; Modifiable \\
DOT_ship_name & Alpha 60 & Enterable; Modifiable \\
timestamp & Enterable; Modifiable \\
Review_date & Long Integer & Enterable; Modifiable \\
Density & Date & Enterable; Modifiable \\
Molecular_wght & Real & Enterable; Modifiable \\
Vapor_pressure & Real & Enterable; Modifiable \\
Boiling_point & Real & Enterable; Modifiable \\
postref & Real & Enterable; Modifiable \\
reserved2 & Long Integer & Enterable; Modifiable \\
reserved3 & Picture & Enterable; Modifiable \\
\hline
\end{tabular}




\begin{tabular}{|l|l|l|}
\hline \hline \multicolumn{2}{|c|}{ Structure: ChemConstituent } \\
\hline ConstituentName & Alpha 80 & Indexed; Mandatory; Enterable; Modifiable \\
Mfg & Alpha 40 & Mandatory; Enterable; Modifiable \\
Chemical_ID & Long Integer & Indexed; Enterable; Modifiable \\
PC_byWeight_max & Real & Enterable; Modifiable \\
PC_byWeight_min & Real & Enterable; Modifiable \\
timestamp & Long Integer & Enterable; Modifiable \\
postref & Long Integer & Enterable; Modifiable \\
\hline
\end{tabular}

\begin{tabular}{|l|l|l|}
\hline \multicolumn{2}{|c|}{ Structure: Chem_keywords } \\
\hline Chemical_IDD & Long Integer & Indexed; Enterable; Modifiable \\
Keyword & Alpha 30 & Enterable; Modifiable \\
timestamp & Long Integer & Enterable; Modifiable \\
postref & Long Integer & Enterable; Modifiable \\
\hline
\end{tabular}

\begin{tabular}{|l|l|l|}
\hline \multicolumn{2}{|c|}{ Structure: Chem_phys_haz } \\
\hline Chemical_ID & Long Integer & Indexed; Enterable; Modifiable \\
Physical_hazard & Alpha 30 & Enterable; Modifiable \\
timestamp & Long Integer & Enterable; Modifiable \\
postref & Long Integer & Enterable; Modifiable \\
\hline
\end{tabular}

\begin{tabular}{|l|l|l|}
\hline \multicolumn{2}{|c|}{ Structure: Chem_health_haz } \\
\hline Chemical_ID & Long Integer & Indexed; Enterable; Modifiable \\
Health_hazard & Alpha 30 & Enterable; Modifiable \\
timestamp & Long Integer & Enterable; Modifiable \\
postref & Long Integer & Enterable; Modifiable \\
\hline
\end{tabular}




\begin{tabular}{|l|l|l|}
\hline \multicolumn{2}{|c|}{ Structure: Chem_reg_cat } \\
\hline Chemical_ID D & Long Integer & Indexed; Enterable; Modifiable \\
Reg_category & Alpha 30 & Enterable; Modifiable \\
timestamp & Long Integer & Enterable; Modifiable \\
_postref & Long Integer & Enterable; Modifiable \\
\hline
\end{tabular}

\begin{tabular}{|l|l|l|}
\hline \multicolumn{2}{|c|}{ Structure: ChemControlArea } \\
\hline CCA_code & Alpha 15 & Indexed; Unique; Mandatory; Enterable \\
CCA_name & Alpha 80 & Enterable; Modifiable \\
CCA_mgremp_no & Elpha 10 & Enterable; Modifiable \\
Admin_org_ID & Long Integer & Enterable; Modifiable \\
Remarks & Text & Enterable; Modifiable \\
timestamp & Long Integer & Enterable; Modifiable \\
postref & Long Integer & Enterable; Modifiable \\
\hline
\end{tabular}

\begin{tabular}{|l|l|l|}
\hline \multicolumn{2}{|c|}{ Structure: CCA_Locations } \\
\hline CCA_code & Alpha 15 & Indexed; Enterable; Modifiable \\
Building & Alpha 6 & Enterable; Modifiable \\
Location & Alpha 40 & Enterable; Modifiable \\
Remarks & Text & Enterable; Modifiable \\
timestamp & Enterable; Modifiable \\
Room & Long Integer & Enterable; Modifiable \\
Mail_stop & Alpha 10 & Enterable; Modifiable \\
postret & Alpha 10 & Enterable; Modifiable \\
\hline
\end{tabular}




\begin{tabular}{|l|l|l|}
\hline \hline \multicolumn{2}{|c|}{ Structure: People } \\
\hline LLNL_emp_no & Alpha 10 & Indexed; Unique; Mandatory; Enterable \\
Lastname & Alpha 40 & Enterable; Modifiable \\
Firstname & Alpha 40 & Enterable; Modifiable \\
Ml & Alpha 5 & Enterable; Modifiable \\
Lab_phone & Alpha 10 & Enterable; Modifiable \\
Mail_stop & Alpha 10 & Enterable; Modifiable \\
Bldg & Alpha 6 & Enterable; Modifiable \\
Room & Elpha 6 & Enterable; Modifiable \\
Payroll_acct & Alpha 4 & Enterable; Modifiable \\
timestamp & Long Integer & Enterable; Modifiable \\
postref & Long Integer & Enterable; Modifiable \\
\hline
\end{tabular}

\begin{tabular}{|l|l|l|}
\hline \multicolumn{2}{|c|}{ Structure: Admin_org } \\
\hline Admin_org_ID & Long Integer & Indexed; Unique; Mandatory; Enterable \\
Admin_org_name & Alpha 80 & Enterable; Modifiable \\
Directorate & Alpha 80 & Enterable; Modifiable \\
Department & Alpha 80 & Enterable; Modifiable \\
Division & Alpha 80 & Enterable; Modifiable \\
Section & Alpha 80 & Enterable; Modifiable \\
LLNLGroup & Alpha 80 & Enterable; Modifiable \\
Program & Alpha 80 & Enterable; Modifiable \\
timestamp & Long Integer & Enterable; Modifiable \\
Payroll_acct & Enterable; Modifiable \\
postret & Llpha 4 & Enterable; Modifiable \\
\hline
\end{tabular}

\begin{tabular}{|l|l|l|}
\hline \multicolumn{2}{|c|}{ Structure: PC_status } \\
\hline PC_status & Alpha 12 & Enterable; Modifiable \\
timestamp & Long Integer & Enterable; Modifiable \\
postret & Long Integer & Enterable; Modifiable \\
\hline
\end{tabular}




\begin{tabular}{|l|l|l|}
\hline \multicolumn{2}{|c|}{ Structure: Qty_units } \\
\hline $\begin{array}{l}\text { Qty_units } \\
\text { timestamp } \\
\text { postref }\end{array}$ & Alpha 10 & Enterable; Modifiable \\
\hline
\end{tabular}

\begin{tabular}{|l|l|l|}
\hline \multicolumn{2}{|c|}{ Structure: Material_use } \\
\hline $\begin{array}{l}\text { Material_use } \\
\text { timestamp } \\
\text { postref }\end{array}$ & Alpha 30 & Enterable; Modifiable \\
& Long Integer & Enterable; Modifiable \\
\hline
\end{tabular}

\begin{tabular}{|l|l|l|}
\hline \multicolumn{2}{|c|}{ Structure: Container_type } \\
\hline $\begin{array}{l}\text { Container_type } \\
\text { timestamp } \\
\text { postref }\end{array}$ & Alpha 10 & Enterable; Modifiable \\
\hline
\end{tabular}

\begin{tabular}{|l|l|l|}
\hline \multicolumn{2}{|c|}{ Structure: Synonym_type } \\
\hline Synonym_type & Alpha 20 & Enterable; Modifiable \\
timestamp & Long Integer & Enterable; Modifiable \\
postref & Long Integer & Enterable; Modifiable \\
\hline
\end{tabular}

\begin{tabular}{|l|l|l|}
\hline \multicolumn{2}{|c|}{ Structure: Material_type } \\
\hline $\begin{array}{l}\text { Material_type } \\
\text { timestamp } \\
\text { postref }\end{array}$ & Alpha 20 & Enterable; Modifiable \\
& Long Integer & Enterable; Modifiable \\
\hline
\end{tabular}




\begin{tabular}{|l|l|l|}
\hline \multicolumn{2}{|c|}{ Structure: Physical_state } \\
\hline $\begin{array}{l}\text { Physical_state } \\
\text { timestamp } \\
\text { postref }\end{array}$ & Alpha 7 & Enterable; Modifiable \\
& Long Integer & Enterable; Modifiable \\
Long Integer & Enterable; Modifiable \\
\hline
\end{tabular}

\begin{tabular}{|l|l|l|}
\hline \multicolumn{2}{|c|}{ Structure: ChemTrack_class } \\
\hline ChemTrack_class & Alpha 2 & Enterable; Modifiable \\
Description & Alpha 80 & Enterable; Modifiable \\
Remarks & Text & Enterable; Modifiable \\
timestamp & Long Integer & Enterable; Modifiable \\
postref & Long Integer & Enterable; Modifiable \\
\hline
\end{tabular}

\begin{tabular}{|l|l|l|}
\hline \multicolumn{2}{|c|}{ Structure: Hazard_class } \\
\hline Hazard_class & Alpha 2 & Enterable; Modifiable \\
Description & Alpha 80 & Enterable; Modifiable \\
timestamp & Long Integer & Enterable; Modifiable \\
postref & Long Integer & Enterable; Modifiable \\
\hline
\end{tabular}

\begin{tabular}{|l|l|l|}
\hline \multicolumn{2}{|c|}{ Structure: Chem_keywd_tbl } \\
\hline Keyword & Alpha 30 & Enterable; Modifiable \\
timestamp & Long Integer & Enterable; Modifiable \\
postref & Long Integer & Enterable; Modifiable \\
\hline
\end{tabular}




\begin{tabular}{|l|l|l|}
\hline \multicolumn{2}{|c|}{ Structure: Phys_haz_tbl } \\
\hline $\begin{array}{l}\text { Physical_hazard } \\
\text { timestamp } \\
\text { postref }\end{array}$ & Alpha 30 & Enterable; Modifiable \\
\hline
\end{tabular}

\begin{tabular}{|l|l|l|}
\hline \multicolumn{2}{|c|}{ Structure: Health_haz_tbl } \\
\hline Health_hazard & Alpha 30 & Enterable; Modifiable \\
timestamp & Long Integer & Enterable; Modifiable \\
postref & Long Integer & Enterable; Modifiable \\
\hline
\end{tabular}

\begin{tabular}{|l|l|l|}
\hline \multicolumn{2}{|c|}{ Structure: Reg_cat_tbl } \\
\hline $\begin{array}{l}\text { Reg_category } \\
\text { timestamp } \\
\text { postref }\end{array}$ & Alpha 30 & Enterable; Modifiable \\
& Long Integer & Enterable; Modifiable \\
\hline
\end{tabular}




\begin{tabular}{|l|l|l|}
\hline \hline \multicolumn{2}{|c|}{ Structure: TrackingHistory } \\
\hline CT_barcode & Alpha 10 & Enterable; Modifiable \\
Substance_name & Alpha 80 & Enterable; Modifiable \\
Substance_mfg & Alpha 40 & Enterable; Modifiable \\
CCA_Code & Alpha 15 & Enterable; Modifiable \\
Current_qty & Real & Enterable; Modifiable \\
Qty_units & Alpha 10 & Enterable; Modifiable \\
Container_owner & Alpha 10 & Enterable; Modifiable \\
Container_type & Alpha 10 & Enterable; Modifiable \\
PC_status & Alpha 12 & Enterabie; Modifiable \\
Scan_date & Date & Enterable; Modifiable \\
_postref & Long integer & Enterable; Modifiable \\
_unused2 & Picture & Enterable; Modifiable \\
_unused3 & Picture & Enterable; Modifiable \\
tunused4 & Picture & Enterable; Modifiable \\
timestamp & Long Integer & Enterable; Modifiable \\
_unused5 & Picture & Enterable; Modifiable \\
\hline
\end{tabular}

\begin{tabular}{|l|l|l|}
\hline \multicolumn{2}{|c|}{ Structure: StoragePressure } \\
\hline Pressure_code & Alpha 2 & Enterable; Modifiable \\
Description & Alpha 20 & Enterable; Modifiable \\
timestamp & Long Integer & Enterable; Modifiable \\
postret & Long Integer & Enterable; Modifiable \\
\hline
\end{tabular}

\begin{tabular}{|l|l|l|}
\hline \multicolumn{2}{|c|}{ Structure: Storage_temp } \\
\hline Temp_code & Alpha 2 & Enterable; Modifiable \\
Description & Alpha 20 & Enterable; Modifiable \\
timestamp & Long Integer & Enterable; Modifiable \\
postret & Long Integer & Enterable; Modifiable \\
\hline
\end{tabular}




\begin{tabular}{|l|l|l|}
\hline \hline \multicolumn{2}{|c|}{ Structure:_transaction } \\
\hline transaction_no & Long Integer & Indexed; Unique; Enterable \\
post_state & Integer & Enterable; Modifiable \\
post_timestamp & Long Integer & Enterable; Modifiable \\
retcode & Integer & Enterable; Modifiable \\
retmsg & Text & Enterable; Modifiable \\
fail_stmnt_no & Long Integer & Enterable; Modifiable \\
\hline
\end{tabular}

\begin{tabular}{|l|l|l|}
\hline \multicolumn{2}{|c|}{ Structure:_statement } \\
\hline statement_no & Long Integer & Indexed; Unique; Enterable \\
transaction_no & Long Integer & Indexed; Enterable; Modifiable \\
file_no & Integer & Enterable; Modifiable \\
postref_field & Integer & Enterable; Modifiable \\
where_clause & Text & Enterable; Modifiable \\
rec_timestamp & Long Integer & Enterable; Modifiable \\
local_key_val & Long Integer & Enterable; Modifiable \\
local_key_fld & Integer & Enterable; Modifiable \\
\hline
\end{tabular}

\begin{tabular}{|l|l|l|}
\hline \multicolumn{2}{|c|}{ Structure:_SQL_entry } \\
\hline SQL_entry_no & Long Integer & Enterable; Modifiable \\
statement_no & Long Integer & Indexed; Enterable; Modifiable \\
SQL_type & Alpha 6 & Enterable; Modifiable \\
SQL_body & Text & Enterable; Modifiable \\
\hline
\end{tabular}




\begin{tabular}{|l|l|l|}
\hline \multicolumn{2}{|c|}{ Structure: ChemMail } \\
\hline ChemMail_ID & Long Integer & Enterable; Modifiable \\
Msg_subject & Alpha 40 & Enterable; Modifiable \\
From_emp_no & Alpha 10 & Enterable; Modifiable \\
To_CCA_ & Alpha 15 & Enterable; Modifiable \\
CT_barcode & Alpha 10 & Enterable; Modifiable \\
From_CCA & Alpha 15 & Enterable; Modifiable \\
Msg text & Text & Enterable; Modifiable \\
Msg_status & Alpha 10 & Enterable; Modifiable \\
Send_datetime & Date & Enterable; Modifiable \\
User login & Alpha 15 & Enterable; Modifiable \\
timestamp & Long Integer & Enterable; Modifiable \\
_postref & Long Integer & Enterable; Modifiable \\
\hline
\end{tabular}

\begin{tabular}{|l|l|l|}
\hline \multicolumn{2}{|c|}{ Structure: Inventory } \\
\hline CCA_code & Alpha 15 & Enterable; Modifiable \\
CT_barcode & Alpha 10 & Enterable; Modifiable \\
Inventory_date & Date & Enterable; Modifiable \\
Upload_date & Date & Enterable; Modifiable \\
ErrCode & Long Integer & Enterable; Modifiable \\
Error_msg & Text & Enterable; Modifiable \\
PC_status & Alpha 12 & Enterable; Modifiable \\
\hline
\end{tabular}




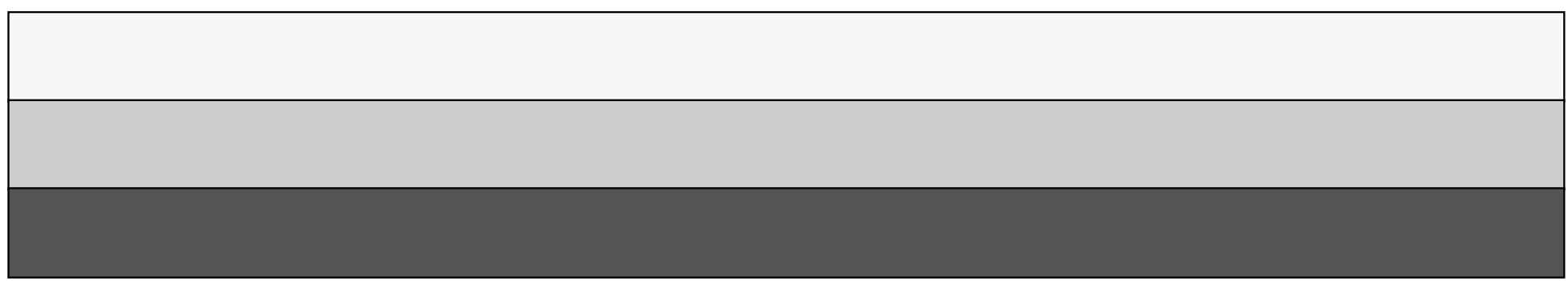

